\title{
Fotoluminescência em amostras policristalinas de galato de magnésio dopado com $\mathrm{Ni}^{2+}$
}

\section{(Photoluminescence of polycrystalline samples of magnesium gallate doped with $\mathrm{Ni}^{2+}$ )}

\author{
L. P. Sosman ${ }^{1}$, R. J. M. da Fonseca ${ }^{1}$, A. Dias Tavares Jr. ${ }^{1}$, T. Abritta ${ }^{2}$ \\ ${ }^{I}$ DEQ, Instituto de Física, UERJ, Rua São Francisco Xavier 524, Rio de Janeiro, RJ 20559-900 \\ ${ }^{2}$ Instituto de Física, UFRJ, C.P. 68528, Rio de Janeiro, RJ 21945-970 \\ rauljose@uerj.br
}

\begin{abstract}
Resumo
São apresentados resultados de fotoluminescência do $\mathrm{MgGa}_{2} \mathrm{O}_{4}: \mathrm{Ni}^{2+}$ nas temperaturas ambiente e de nitrogênio líquido. As bandas observadas são atribuídas às transições proibidas por spin, ${ }^{1} \mathrm{~T}_{2}\left({ }^{1} \mathrm{D}\right) \rightarrow{ }^{3} \mathrm{~T}_{2}\left({ }^{3} \mathrm{~F}\right) \mathrm{e}^{1} \mathrm{~T}_{2}\left({ }^{1} \mathrm{D}\right) \rightarrow{ }^{3} \mathrm{~T}_{1}\left({ }^{3} \mathrm{~F}\right)$, no visível e no infravermelho próximo, respectivamente. Espectros de absorção obtidos a partir de medidas de reflectância difusa exibem bandas associadas às transições ${ }^{3} \mathrm{~A}_{2}\left({ }^{3} \mathrm{~F}\right) \rightarrow{ }^{3} \mathrm{~T}_{1}\left({ }^{3} \mathrm{~F}\right) \mathrm{e}^{3} \mathrm{~A}_{2}\left({ }^{3} \mathrm{~F}\right) \rightarrow{ }^{3} \mathrm{~T}_{2}$ $\left({ }^{3} \mathrm{~F}\right)$ e uma fraca estrutura identificada com a transição ${ }^{3} \mathrm{~A}_{2}\left({ }^{3} \mathrm{~F}\right) \rightarrow{ }^{1} \mathrm{E}\left({ }^{1} \mathrm{D}\right)$. Todas as transições observadas são identificadas com níveis de energia do $\mathrm{Ni}^{2+}$ em sítios octaédricos do galato de magnésio. A partir dos diagramas de energia de Tanabe-Sugano para configuração $\mathrm{d}^{8}$ foram calculados os parâmetros de campo cristalino, $\mathrm{D}_{\mathrm{q}}$ e de repulsão intereletrônica, B e C. Apresentamos também, para efeito de comparação, resultados anteriores dos íons $\mathrm{Cr}^{3+}$ (configuração eletrônica d $\left.\mathrm{d}^{3}\right), \mathrm{Fe}^{3+}\left(\mathrm{d}^{5}\right)$ e $\mathrm{Co}^{2+}\left(\mathrm{d}^{7}\right)$ nesta rede hospedeira para amostras preparadas com o mesmo método cerâmico. Palavras-chave: fotoluminescência, absorção, material cerâmico, metais de transição.
\end{abstract}

Abstract

The photoluminescence of $\mathrm{MgGa} \mathrm{O}_{4}: \mathrm{Ni}^{2+}$ are presented in this article. The observed bands are attributed to the ${ }^{I} T_{2}\left({ }^{1} D\right) \rightarrow{ }^{3} T_{2}\left({ }^{\beta} F\right)$ and ${ }^{1} T_{2}\left({ }^{1} D\right) \rightarrow$ $\left.{ }^{3} T_{1} \beta F\right)$ spin-forbidden transitions and located in the visible and infrared regions, respectively. Absorption spectra obtained from diffuse reflectance measurements show bands associated with ${ }^{3} A_{2}\left({ }^{\beta} F\right) \rightarrow{ }^{3} T_{1}\left({ }^{\beta} F\right)$ and ${ }^{3} A_{2}\left({ }^{\beta} F\right) \rightarrow{ }^{3} T_{2}\left({ }^{\beta} F\right)$ transitions and a weak structure assigned to ${ }^{3} A_{2}\left({ }^{\beta} F\right) \rightarrow{ }^{1} E$ ( $\left.{ }^{\prime} D\right)$ transition. All the observed transitions are identified with energy levels of $\mathrm{Ni}^{2+}$ in octahedral sites of magnesium gallate. From the TanabeSugano energy diagrams for a $d^{8}$ configuration, the crystal field $D q$ and interelectronic repulsion $B$ and C parameters were calculated. We also present, for comparison, previous results of $\mathrm{Cr}^{3+}$ (d electronic configuration), $\mathrm{Fe}^{3+}\left(d^{5}\right)$ and $\mathrm{Co}^{2+}\left(d^{7}\right)$ ions in this host lattice for samples prepared with the same ceramic method.

Keywords: photoluminescence, absorption, ceramic materials, transition metals.

\section{INTRODUÇÃO}

Íons de metais de transição quando introduzidos como impurezas substitucionais em materiais isolantes hospedeiros podem apresentar largas bandas de absorção e de luminescência no visível e no infravermelho próximo. As propriedades luminescentes dos metais de transição são úteis para diversas aplicações, que variam de materiais ativos para laser de estado sólido a temperatura ambiente até a fabricação de folhas de papel especial $[1,2]$. $\mathrm{O} \mathrm{Ni}^{2+}$ (configuração eletrônica d ${ }^{8}$ ) é um íon de metal de transição cuja luminescência não é tão freqüente quanto a do $\mathrm{Co}^{2+}\left(\mathrm{d}^{7}\right)$, $\mathrm{Cr}^{3+}\left(\mathrm{d}^{3}\right)$ ou ainda a do $\mathrm{Fe}^{3+}\left(\mathrm{d}^{5}\right)$. Este é um fato que justifica o nosso interesse na produção e no estudo de amostras contendo a impureza $\mathrm{Ni}^{2+}$. Além disso, os íons $\mathrm{Co}^{2+}, \mathrm{Cr}^{3+}$ e $\mathrm{Fe}^{3+}$ em amostras policristalinas de $\mathrm{MgGa}_{2} \mathrm{O}_{4}$ têm sido estudados recentemente [3-7] e este trabalho busca também estender este estudo com as propriedades espectroscópicas do $\mathrm{MgGa}_{2} \mathrm{O}_{4}: \mathrm{Ni}^{2+}$.

A rede hospedeira $\mathrm{MgGa}_{2} \mathrm{O}_{4}$ é formada por íons de camadas fechadas. $\mathrm{O}$ íon dopante $\mathrm{Ni}^{2+}$ tem a valência e o raio atômico $\left(\mathrm{r}_{\mathrm{Ni}}{ }^{2+}=0,7 \AA\right)$ com valores próximos ao do íon que será substituído, o $\mathrm{Mg}^{2+}\left(\mathrm{r}_{\mathrm{Mg}}{ }^{2+}=0,66 \AA\right.$ ) , o que facilita a substituição no processo de dopagem. Entretanto não é rara a ocorrência de redes hospedeiras que não satisfaçam estas exigências. Neste caso a diferença de valência, ou de raios iônicos, é compensada por novas posições dos íons da rede durante o processo de síntese. Quando a impureza é inserida na rede, os níveis de energia são desdobrados e muitos destes novos estados dependem fortemente do potencial gerado pelos ânions vizinhos (campo cristalino). Esta dependência, associada com pequenas diferenças estruturais no sítio de ocupação do dopante, origina as largas bandas observadas, denominadas bandas vibracionais. Transições 
luminescentes deste íon têm sido apresentadas na literatura, para alguns materiais óxidos. Para o $\mathrm{MgO}$, foi verificada uma banda de emissão em baixas temperaturas situada na região do infravermelho, mais precisamente no intervalo de comprimento de onda entre $1316 \mathrm{~nm}$ e $1409 \mathrm{~nm}[8,9]$. Por sua vez, no $\mathrm{Gd}_{3} \mathrm{Ga}_{5} \mathrm{O}_{12}$ e $\mathrm{CaY}_{2} \mathrm{Mg}_{2} \mathrm{Ge}_{3} \mathrm{O}_{12}$ estas bandas encontram-se nas regiões de $1434 \mathrm{~nm}$ a $1520 \mathrm{~nm}$ e de $1410 \mathrm{~nm}$ a $1520 \mathrm{~nm}$, respectivamente [10-12]. Para os dois primeiros materiais, a oscilação laser ocorre em temperaturas inferiores a $100 \mathrm{~K}$, enquanto para o último material a oscilação laser não foi observada. Em todos os casos, a emissão laser na temperatura ambiente não ocorre, pois a absorção do estado fundamental superpõe com o alcance espectral de emissão. Ainda nestes materiais, os sítios ocupados pelo $\mathrm{Ni}^{2+}$ possuem uma simetria octaédrica e, para o $\mathrm{Gd}_{3} \mathrm{Ga}_{5} \mathrm{O}_{12}$, ocorre ainda um processo de compensação de carga visto que o $\mathrm{Ga}$ é um íon trivalente.

\section{MATERIAIS E MÉTODOS}

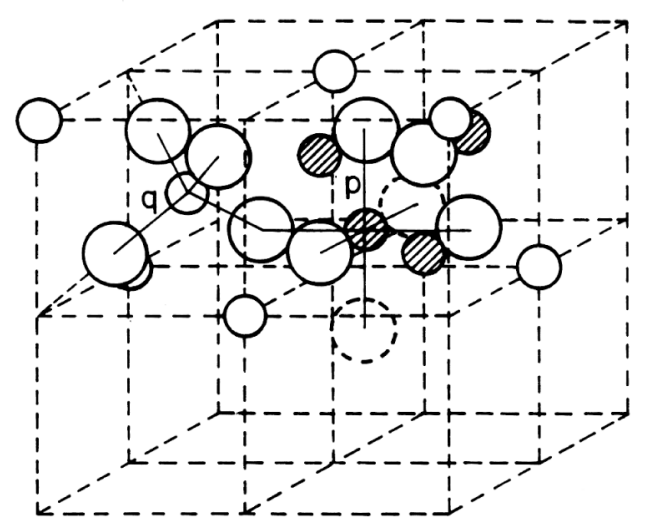

Figura 1: Estrutura espinel. Os cátions $\mathrm{Mg}^{2+} \mathrm{e} \mathrm{Ga}^{3+}$ são distribuídos pelos sítios coordenados octaedricamente (p) e tetraedricamente (q) pelos ânions $\mathrm{O}^{2-}$.

[Figure 1: Spinel structure. The $\mathrm{Mg}^{2+}$ and $\mathrm{Ga}^{3+}$ cations are distributed on octahedrally (p) and tetrahedrally (q) sites coordinated by $\mathrm{O}^{2-}$ anions.]

As amostras policristalinas usadas nesse trabalho foram produzidas por reação de estado sólido entre os óxidos $\mathrm{MgO}, \beta-\mathrm{Ga}_{2} \mathrm{O}_{3}$ e uma quantidade conveniente de $\mathrm{Ni}(\mathrm{OH})_{2}$. $\mathrm{O}$ tratamento térmico foi realizado a $1400{ }^{\circ} \mathrm{C} \mathrm{em} \mathrm{um}$ período de 24 horas. O galato de magnésio tem estrutura espinel parcialmente invertida [13]. Em um artigo anterior [14], a estrutura espinel foi descrita como uma classe de materiais $\mathrm{AB}_{2} \mathrm{O}_{4}$ nos quais existem duas posições para os cátions $\mathrm{A}^{2+} \mathrm{e} \mathrm{B}^{3+}$. No espinel normal, os íons de oxigênio são dispostos em um arranjo cúbico de face centrada com os cátions metálicos nos interstícios (Fig. 1). A célula unitária contém 32 oxigênios com 96 interstícios, onde 32 destes sítios são coordenados octaedricamente (íons B) e os outros
64 são coordenados tetraedricamente (íons A). No espinel intermediário ou parcialmente invertido, os cátions $\mathrm{A}^{2+}$ and $\mathrm{B}^{3+}$ são distribuídos randomicamente sobre os sítios tetraédricos e octaédricos. Os espinéis invertidos são aqueles nos quais os sítios tetraédricos são ocupados por cátions $\mathrm{B}^{3+}$ e os sítios octaédricos são ocupados randomicamente por cátions $\mathrm{A}^{2+}$ e $\mathrm{B}^{3+}$. A ocupação dos sítios octaédricos por cátions divalentes perturba a rede. $\mathrm{O}$ espinel $\mathrm{MgGa}_{2} \mathrm{O}_{4}$ pertence ao grupo espacial cúbico $\mathrm{O}_{\mathrm{h}}{ }^{7}(\mathrm{~F} d 3 \mathrm{~m})$ com simetria pontual $\mathrm{T}_{\mathrm{d}}(\overline{4} 3 \mathrm{~m})$ para os sítios tetraédricos e $\mathrm{D}_{3 \mathrm{~d}}(\overline{3} \mathrm{~m})$ para os octaédricos e com parâmetro de célula $a=8.286$ Å. A ocupação determinada para a população de $\mathrm{Mg}^{2+}$ foi de $16 \pm 2 \%$ em sítios tetraédricos e de $81 \pm 1 \%$ em sítios octaédricos [13-15].

A formação do composto foi verificada por difração de raios $\mathrm{X}$ usando um difratômetro de pó Siemens tipo $\mathrm{F}$ equipado com um tubo de cobalto e monocromador. Os dados obtidos foram comparados com aqueles da literatura, mostrando a formação do composto desejado [16]. Os dados ópticos foram obtidos usando um espectrômetro Spex, modelo 1702, para varrer a emissão da amostra. Para as medidas de luminescência empregamos como fonte de excitação um laser de hélio-neônio $(632.8 \mathrm{~nm})$ com $15 \mathrm{~mW}$ de potência e filtros ópticos para cortar possíveis linhas de interferência de ordem superior. O feixe de excitação foi modulado em amplitude por um modulador eletromecânico PAR 191 com velocidade variável. O detector utilizado nestas medidas foi um RCA 7102 refrigerado, conectado a um amplificador-retificador sensível a fase EGG 5209, interfaceado a um PC para a armazenagem e tratamento de dados. As medidas de absorção foram feitas com uma lâmpada de tungstênio de $100 \mathrm{~W}$. Os valores de tempo de vida do estado excitado foram obtidos pelo método de deslocamento de fase.

\section{RESULTADOS E DISCUSSÃO}

Em simetria octaédrica, o desdobramento dos níveis de energia da configuração eletrônica $\mathrm{d}^{8}$ é descrito pelo diagrama de Tanabe-Sugano [17], mostrado na Fig. 2. Para esta configuração, o termo de menor energia ${ }^{3} \mathrm{~F}$ desdobra-se no nível fundamental ${ }^{3} \mathrm{~A}_{2}$ e em dois níveis excitados, ${ }^{3} \mathrm{~T}_{2} \mathrm{e}^{3} \mathrm{~T}_{1}$. $\mathrm{O}$ termo de energia imediatamente superior ${ }^{1} \mathrm{D}$, desdobra-se em dois níveis, ${ }^{1} \mathrm{E} \mathrm{e}^{1} \mathrm{~T}_{2}$. Para sistemas com campo cristalino fraco o primeiro estado excitado é o ${ }^{3} \mathrm{~T}_{2}$, enquanto que para sistemas de campo cristalino intermediário ou forte o estado excitado inferior é o ${ }^{1} \mathrm{E}\left({ }^{1} \mathrm{D}\right)$.

$\mathrm{O}$ espectro de absorção do $\mathrm{MgGa}_{2} \mathrm{O}_{4}$ com $0,5 \%$ de $\mathrm{Ni}^{2+}$ obtido a partir da reflectância difusa a temperatura ambiente é mostrado na Fig. 3.

Neste espectro observamos uma larga e intensa banda com baricentro em $618 \mathrm{~nm}$ e uma outra banda menos intensa com máximo de intensidade em $992 \mathrm{~nm}$. A banda de menor energia corresponde à transição permitida por spin ${ }^{3} \mathrm{~A}_{2}$ 


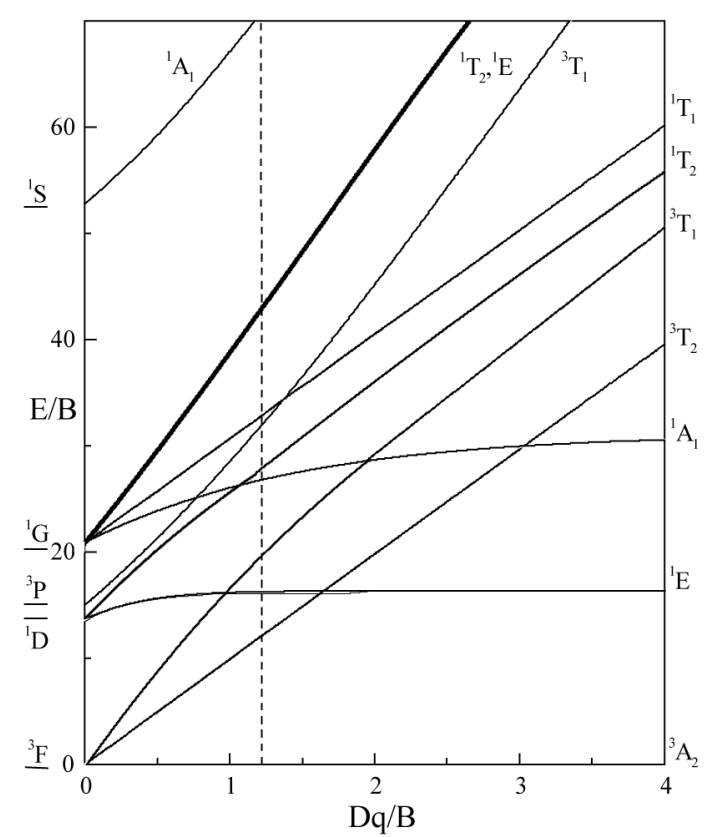

Figura 2: Diagrama de Tanabe-Sugano para a configuração d $\mathrm{d}^{8} \mathrm{em}$ sítio octaédrico. A linha pontilhada mostra a relação $\mathrm{Dq} / \mathrm{B}=1,22$ obtida neste trabalho.

[Figure 2: Tanabe-Sugano diagram to $d^{8}$ configuration in octahedral sites. The dashed line shows the relation $D q / B=1.22$ obtained in this work.]

$\left({ }^{3} \mathrm{~F}\right) \rightarrow{ }^{3} \mathrm{~T}_{2}\left({ }^{3} \mathrm{~F}\right)$ e a banda de maior energia é atribuída à transição ${ }^{3} \mathrm{~A}_{2}\left({ }^{3} \mathrm{~F}\right) \rightarrow{ }^{3} \mathrm{~T}_{1}\left({ }^{3} \mathrm{~F}\right)$. Além dessas duas bandas observamos também uma fraca estrutura em torno de 774 $\mathrm{nm}$, identificada com a transição proibida por spin ${ }^{3} \mathrm{~A}_{2}$ $\left({ }^{3} \mathrm{~F}\right) \rightarrow{ }^{1} \mathrm{E}\left({ }^{1} \mathrm{D}\right)$. No espectro observamos o início de uma terceira banda, que é descrita na literatura [18-19] como referente à transição ${ }^{3} \mathrm{~A}_{2}\left({ }^{3} \mathrm{~F}\right) \rightarrow{ }^{3} \mathrm{~T}_{1}\left({ }^{3} \mathrm{P}\right)$. Esta banda não foi observada porque o aparato experimental disponível é limitado nesta região espectral. A partir das posições em energia dos máximos de intensidade das bandas do espectro de absorção, podemos calcular os parâmetros de campo cristalino Dq e de Racah B e C, utilizando as seguintes expressões (equações A, B, C e D) fornecidas pelas matrizes de energia de Tanabe-Sugano para a configuração $d^{8}[17]$ :
Os valores obtidos foram $\mathrm{Dq}=1008 \mathrm{~cm}^{-1}, \mathrm{~B}=824$ $\mathrm{cm}^{-1}$ e $\mathrm{C}=3355 \mathrm{~cm}^{-1}$. A relação $\mathrm{Dq} / \mathrm{B}=1,22$ é utilizada para compararmos as posições em energia das bandas de absorção observadas com a relação entre as energias dos níveis fornecidos pelo diagrama de Tanabe-Sugano para a configuração $d^{8}$. Por este diagrama, quando localizamos o valor de $\mathrm{Dq} / \mathrm{B}=1,22$ (linha pontilhada na Fig. 2), a razão ${ }^{3} \mathrm{~T}_{1}\left({ }^{3} \mathrm{~F}\right) /{ }^{3} \mathrm{~T}_{2}\left({ }^{3} \mathrm{~F}\right)$ é igual a 1,61 . A partir dos espectros, o nível ${ }^{3} \mathrm{~T}_{1}\left({ }^{3} \mathrm{~F}\right)$ está em $16181 \mathrm{~cm}^{-1}(618 \mathrm{~nm})$, enquanto o nível ${ }^{3} \mathrm{~T}_{2}$ $\left({ }^{3} \mathrm{~F}\right)$ tem máximo em $10081 \mathrm{~cm}^{-1}(992 \mathrm{~nm})$, o que fornece uma relação em energia entre estes níveis de 1,61, ou seja, idêntica ao valor obtido teoricamente. Com as matrizes de Tanabe-Sugano (equações A-D) e com os valores obtidos experimentalmente para Dq, B e C, a posição em comprimento de onda encontrada para a transição ${ }^{3} \mathrm{~A}_{2}\left({ }^{3} \mathrm{~F}\right)$ $\rightarrow{ }^{3} \mathrm{~T}_{1}\left({ }^{3} \mathrm{P}\right)$ seria $379 \mathrm{~nm}$. Pelo diagrama de Tanabe-Sugano

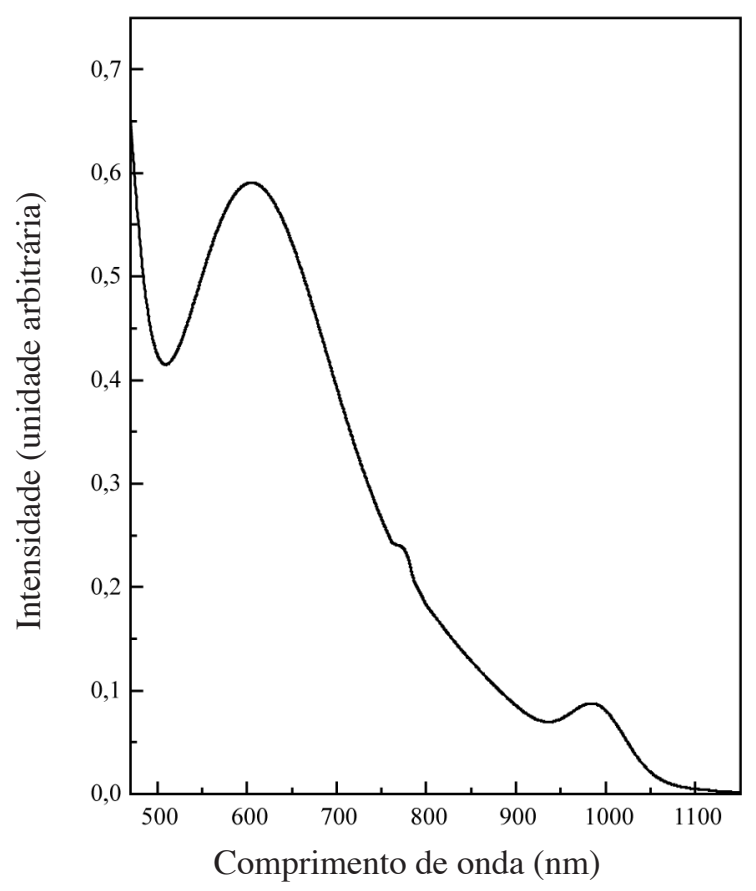

Figura 3: Espectro de absorção do $\mathrm{MgGa}_{2} \mathrm{O}_{4}$ com $0,5 \%$ de $\mathrm{Ni}^{2+}$ obtido a partir da reflectância difusa a temperatura ambiente.

[Figure 3: Absorption spectrum of $\mathrm{MgGa}_{2} \mathrm{O}_{4}$ with $0.5 \%$ of $\mathrm{Ni}^{2+}$ obtained from diffuse reflactance data at room temperature.]

$$
\begin{aligned}
& \left.{ }_{A_{2}}\left({ }^{3} F\right) \rightarrow{ }^{3} T_{2}{ }^{3} F\right)=10 D q \\
& \left.\stackrel{3}{A}_{2}{ }^{3} F\right) \rightarrow{ }^{3} T_{1}\left({ }^{3} P\right)=7,5 B+15 D q+\frac{1}{2}\left[(9 B-10 D q)^{2}+144 B^{2}\right]^{1 / 2} \\
& { }_{A}^{3}\left({ }^{3} F\right) \rightarrow{ }^{3} T_{1}\left({ }^{3} F\right)=7,5 B+15 D q-\frac{1}{2}\left[(9 B-10 D q)^{2}+144 B^{2}\right]^{1 / 2} \\
& 3_{2}\left({ }^{3} F\right) \rightarrow{ }^{1} E\left({ }^{1} D\right)=2 C+10 D q+\frac{17 B}{2}-\frac{1}{2}\left[400 D q^{2}+40 D q B+49 B^{2}\right]^{1 / 2}
\end{aligned}
$$




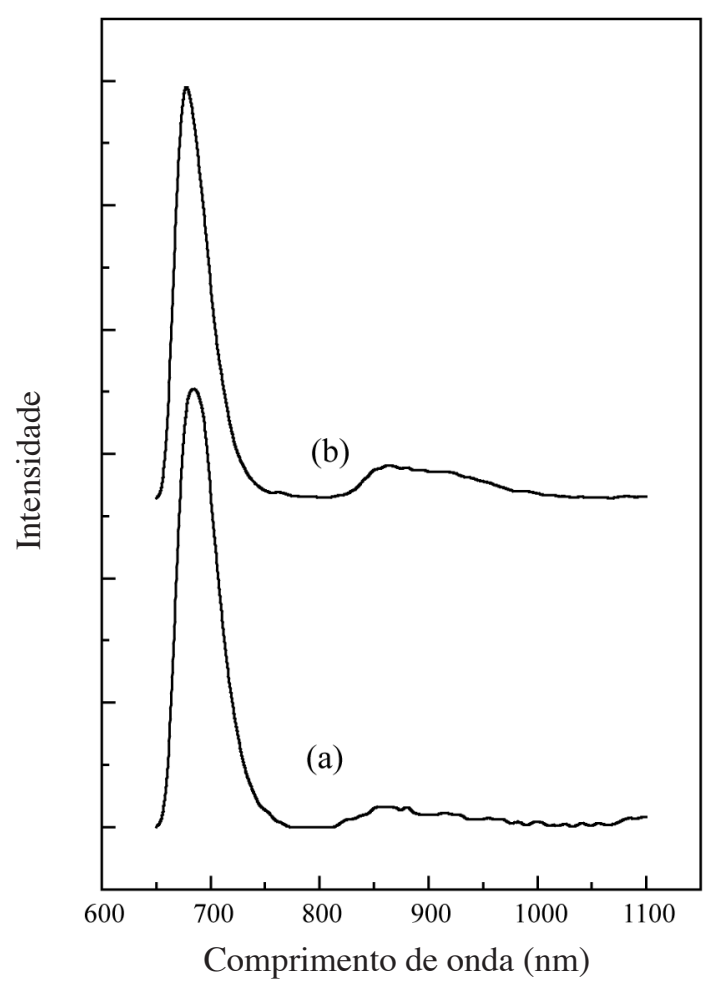

Figura 4: Espectros de luminescência do $\mathrm{MgGa}_{2} \mathrm{O}_{4}$ com $0,5 \%$ de $\mathrm{Ni}^{2+}$ a (a) temperatura ambiente (b) $77 \mathrm{~K}$.

[Figure 4:Luminescence spectra of $\mathrm{MgGa}_{2} \mathrm{O}_{4}$ with $0.5 \%$ of $\mathrm{Ni}^{2+}$ at (a) room temperature and (b) $77 \mathrm{~K}$.]

a posição desta banda seria ajustada em $387 \mathrm{~nm}$. Fazendo a conversão em energia, em unidades de $\mathrm{cm}^{-1}$, o erro entre a estimativa experimental e o ajuste teórico é da ordem de $2 \%$.

Os espectros de fotoluminescência da amostrasão exibidos na Fig.4. Na temperatura ambiente, Fig.4 (a), observamos uma banda intensa na região do vermelho, centrada em 685 $\mathrm{nm}$, que se estende até o infravermelho próximo. Esta banda é associada à transição ${ }^{1} \mathrm{~T}_{2}\left({ }^{1} \mathrm{D}\right) \rightarrow{ }^{3} \mathrm{~T}_{2}\left({ }^{3} \mathrm{~F}\right)$. A hipótese é que o íon é excitado até o nível ${ }^{3} \mathrm{~T}_{1}\left({ }^{3} \mathrm{P}\right)$ a partir do qual decai não-radiativamente para o estado imediatamente inferior ${ }^{1} \mathrm{~T}_{2}\left({ }^{1} \mathrm{D}\right)$. Observamos também uma outra banda muito mais fraca e larga, com máximo de intensidade em torno de 878 $\mathrm{nm}$, correspondendo à transição ${ }^{1} \mathrm{~T}_{2}\left({ }^{1} \mathrm{D}\right) \rightarrow{ }^{3} \mathrm{~T}_{1}\left({ }^{3} \mathrm{~F}\right)$. Na Fig. 4 (b) é mostrado o espectro de fotoluminescência a $77 \mathrm{~K}$. As atribuições das transições observadas são idênticas àquelas feitas para o espectro a temperatura ambiente. A diferença percebida entre os espectros é o suave deslocamento das bandas para a região de maior energia. A transição ${ }^{1} \mathrm{~T}_{2}$ $\left({ }^{1} \mathrm{D}\right) \rightarrow{ }^{3} \mathrm{~T}_{2}\left({ }^{3} \mathrm{~F}\right)$ é localizada agora em $677 \mathrm{~nm}$, enquanto a transição ${ }^{1} \mathrm{~T}_{2}\left({ }^{1} \mathrm{D}\right) \rightarrow{ }^{3} \mathrm{~T}_{1}\left({ }^{3} \mathrm{~F}\right)$ tem máximo de energia em $863 \mathrm{~nm}$. Quando convertidos em energia, o deslocamento da transição ${ }^{1} \mathrm{~T}_{2}\left({ }^{1} \mathrm{D}\right) \rightarrow{ }^{3} \mathrm{~T}_{2}\left({ }^{3} \mathrm{~F}\right)$ entre a temperatura ambiente e $77 \mathrm{~K}$ é de $172 \mathrm{~cm}^{-1}$, enquanto que o deslocamento da transição ${ }^{1} \mathrm{~T}_{2}\left({ }^{1} \mathrm{D}\right) \rightarrow{ }^{3} \mathrm{~T}_{1}\left({ }^{3} \mathrm{~F}\right)$ é de $197 \mathrm{~cm}^{-1}$. Esta diferença pode ser explicada pela largura das bandas de menor energia assim como pelo fato destas serem bastante fracas, dificultando a identificação do baricentro. Os tempos de vida medidos para a transição ${ }^{1} \mathrm{~T}_{2}\left({ }^{1} \mathrm{D}\right) \rightarrow{ }^{3} \mathrm{~T}_{2}\left({ }^{3} \mathrm{~F}\right)$ a temperatura ambiente e a $77 \mathrm{~K}$ foram da ordem de 1,3 ms. Este valor é esperado para sistemas óxidos dopados com $\mathrm{Ni}^{2+} \mathrm{e}$ a independência do tempo de vida radiativo com a temperatura nesta região já foi observada anteriormente [20,21]. Portanto, a eficiência quântica a temperatura ambiente, $\gamma$, estimada pela razão $\gamma=$ $\tau_{300 K} / \tau_{77 \mathrm{~K}}[1]$ é próxima da unidade. O método utilizado para a obtenção do tempo de vida radiativo foi o deslocamento de fase, o qual apresenta um erro da ordem de $10 \%$.

A rede $\mathrm{MgGa}_{2} \mathrm{O}_{4}$ foi utilizada anteriormente como hospedeira dos íons $\mathrm{Cr}^{3+}$ (configuração eletrônica $\mathrm{d}^{3}$ ), $\mathrm{Fe}^{3+}\left(\mathrm{d}^{5}\right)$ e $\mathrm{Co}^{2+}\left(\mathrm{d}^{7}\right)$, sendo todas as amostras preparadas com o mesmo método, descrito neste trabalho [3-5]. As preocupações principais quando se trabalha no preparo de amostras é obter a rede desejada evitando-se tanto quanto possível a formação de fases espúrias e, além disso, garantir que não ocorra contaminação com outras impurezas. Foi mencionado anteriormente que o $\mathrm{Ni}^{2+}$ é um íon cuja luminescência é menos freqüente do que a do $\mathrm{Co}^{2+}$, do $\mathrm{Fe}^{3+}$ ou, principalmente, a do $\mathrm{Cr}^{3+}$. Com isso, apresentamos na Fig. 5 os espectros de luminescência a $77 \mathrm{~K}$ da amostra dopada com (a) $\mathrm{Ni}^{2+}$, (b) $\mathrm{Cr}^{3+}$, (c) $\mathrm{Co}^{2+}$ e (d) $\mathrm{Fe}^{3+}$. Para o íon $\mathrm{Cr}^{3+}$ em sítio octaédrico, a banda exibida na figura, localizada em $707 \mathrm{~nm}$, foi associada à transição ${ }^{2} \mathrm{E}\left({ }^{2} \mathrm{G}\right) \rightarrow{ }^{4} \mathrm{~A}_{2}\left({ }^{4} \mathrm{~F}\right)$ com tempo de vida radiativo de $5,5 \mathrm{~ms}$. A banda do $\mathrm{Co}^{2+}$ tem o baricentro

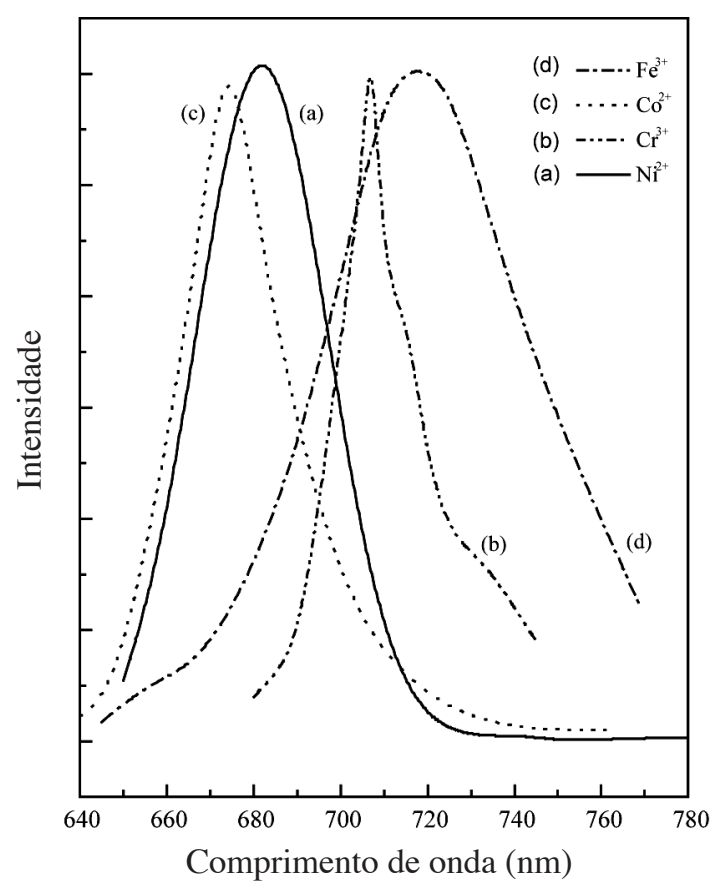

Figura 5: Espectros de luminescência a $77 \mathrm{~K}$ do $\mathrm{MgGa}_{2} \mathrm{O}_{4}$ com (a) $\mathrm{Ni}^{2+}$, (b) $\mathrm{Cr}^{3+}$, (c) $\mathrm{Co}^{2+}$ e (d) $\mathrm{Fe}^{3+}$.

[Figura 5: Luminescence spectra of $\mathrm{MgGa}_{2} \mathrm{O}_{4}$ at $77 \mathrm{~K}$ with (a) $\mathrm{Ni}^{2+}$, (b) $\mathrm{Cr}^{3+}$, (c) $\mathrm{Co}^{2+}$ and (d) $\mathrm{Fe}^{3+}$. 
em $675 \mathrm{~nm}$, tempo de vida de $4 \mu$ s e foi identificada com a transição ${ }^{4} \mathrm{~T}_{1}\left({ }^{4} \mathrm{P}\right) \rightarrow{ }^{4} \mathrm{~A}_{2}\left({ }^{4} \mathrm{~F}\right)$. Por último, a banda centrada em $720 \mathrm{~nm}$ corresponde à transição ${ }^{4} \mathrm{~T}_{1}\left({ }^{4} \mathrm{G}\right) \rightarrow$ ${ }^{6} \mathrm{~A}_{1}\left({ }^{6} \mathrm{~S}\right)$ com tempo de vida de $4 \mathrm{~ms}$. Tanto para o $\mathrm{Co}^{2+}$ quanto para o $\mathrm{Fe}^{3+}$, a análise dos resultados indicou que os íons impureza ocupavam sítios de simetria tetraédrica na rede hospedeira. As distâncias em energia entre a banda do $\mathrm{Ni}^{2+}$ e as bandas do $\mathrm{Cr}^{3+}, \mathrm{Co}^{2+}$ e $\mathrm{Fe}^{3+}$ são dadas respectivamente por $623 \mathrm{~cm}^{-1}, 44 \mathrm{~cm}^{-1}$ e $882 \mathrm{~cm}^{-1}$. Embora a distância em energia entre as transições do $\mathrm{Co}^{2+}$ e do $\mathrm{Ni}^{2+}$ seja relativamente pequena, a diferença na ordem de grandeza entre os tempos de vida mostra que uma das transições é permitida (caso do cobalto divalente) e a outra é proibida (caso do níquel). Assim sendo, pelo formato das bandas, pela distância em energia entre elas, e pelo tempo de vida de cada estado excitado, pode-se perceber claramente que não existe evidência de contaminação por qualquer dos outros íons na amostra de $\mathrm{Ni}^{2+}$.

\section{CONCLUSÕES}

Neste trabalho investigamos as propriedades ópticas do $\mathrm{Ni}^{2+}$ no $\mathrm{MgGa}_{2} \mathrm{O}_{4}$ a temperatura ambiente e a $77 \mathrm{~K}$. Os espectros de luminescência apresentam duas bandas, uma intensa na região do vermelho, associada à transição ${ }^{1} \mathrm{~T}_{2}\left({ }^{1} \mathrm{D}\right) \rightarrow{ }^{3} \mathrm{~T}_{2}\left({ }^{3} \mathrm{~F}\right)$

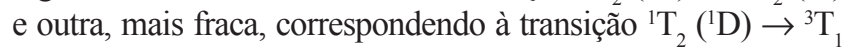
$\left({ }^{3} \mathrm{~F}\right)$. $\mathrm{O}$ formato das bandas e os tempos de vida permanecem constantes para ambas as temperaturas. A partir das bandas de absorção, os parâmetros de energia obtidos foram $\mathrm{Dq}=1008$ $\mathrm{cm}^{-1}, \mathrm{~B}=824 \mathrm{~cm}^{-1} \mathrm{e} \mathrm{C}=3355 \mathrm{~cm}^{-1}$, os quais são valores típicos do $\mathrm{Ni}^{2+}$ em sistemas óxidos com coordenação octaédrica. A relativa simplicidade na obtenção do composto, a alta eficiência quântica da transição mais intensa e as largas bandas de emissão observadas mostram que esse sistema é bastante conveniente para a utilização como meio ativo sintonizável nas regiões do visível e do infravermelho próximo.

\section{AGRADECIMENTOS}

Os autores agradecem a FAPERJ e FINEP pelo suporte financeiro.

\section{REFERÊNCIAS}

[1] S. Kück, Appl. Phys. 72 (2001) 515.

[2] W. K. Zawawy, M. M. Ibrahim, M. G. El-Meligy, Tappi J. 4 (2004) 3.

[3] L. P. Sosman, T. Abritta, Sol. State Commun. 82 (1992) 801.

[4] L. P. Sosman, T. Abritta, O. Nakamura, M. M. F. D’Aguiar Neto, Phys. Stat. Sol. (a) 147 (1995) k107.

[5] L. P. Sosman, A. Dias Tavares Jr., P. S. Silva, T. Abritta, Phys. Stat. Sol. (a) 176 (1999) 1085.

[6] L. P. Sosman, A. Dias Tavares Jr., T. Abritta, J. Phys. D: Appl. Phys. 33 (2000) L19.

[7] L. P. Sosman, T. Abritta, A. C. Pereira, H. Vargas, Chem. Phys. Letters 227 (1994) 485.

[8] P. F. Moulton, Handbook of Laser Science and Technology I, Lasers and Masers, Ed. N. J. Weber (Chemical Rubber Co., Boca Raton 1982) 60.

[9] R. Moncorgé , T. Benyattou, Phys. Rev. B 37 (1988) 9186.

[10] J. Koëtke, K. Petermann, G. Hüber, J. Lumin. 60-61 (1994) 197.

[11] A. Brenier, C. Madej, Phys. Rev. B 49 (1994) 28.

[12] P. F. Moulton, IEEE J. Quantum Electron. 18 (1982) 1185.

[13] J. E. Weinderborner, N.R. Stemple, Y. Okaya, Acta Cryst. 20 (1966) 761.

[14] J. J. Brown, J. Electrochem. Soc. 3 (1967) 245.

[15] C. W. Hoffman, J. J. Brown, J. Inorg. Nuclear Chem. 30 (1968) 63.

[16] Powder Diffraction File 1994 JCPDS-ICDD 10-113.

[17] Y. Tanabe, S. Sugano, J. Phys. Soc. Japan 9 (1954) 753.

[18] N. V. Kuleshov, V. G. Shcherbytsky, V. P. Mykhailov, S. Kück, J. Koetke, K. Petermann, G. Huber, J. Lumin. 71 (1997) 265.

[19] V. P. Solntsev, E. V. Pestryakov, A. I. Alimpiev, E. G. Tsvetkov, V. N. Matrosov, V. I. Trunov, V. V. Petrov, Opt. Mater. 24 (2003) 519.

[20] S. A. Payne, Phys. Rev. B 41 (1990) 6109.

[21] J. F. Donegan, F. J. Bergin, T. J. Glynn, G. F. Imbusch, J. P. Remeika, J. Lumin. 35 (1986) 57.

(Rec. 03/10/2005, Ac. 09/12/2005) 